\title{
Evaluation of a High-Intensity Green Fluorescent Protein Fluorophage Method for Drug- Resistance Diagnosis in Tuberculosis for Isoniazid, Rifampin, and Streptomycin
}

OPEN ACCESS

Edited by:

Julio Alvarez,

University of Minnesota, USA

Reviewed by:

Kuangnan Xiong,

Health Research, Inc., USA

Raju Mukherjee,

Indian Institute of Science Education

and Research, India

*Correspondence:

Hairong Huang

huanghairong@tb123.org

Specialty section:

This article was submitted to Infectious Diseases,

a section of the journal

Frontiers in Microbiology

Received: 25 August 2015 Accepted: 31 May 2016

Published: 17 June 2016

Citation:

Yu X, Gu Y, Jiang G, Ma Y, Zhao L,

Sun Z, Jain P, O'Donnell M, Larsen M, Jacobs WR Jr and Huang H (2016)

Evaluation of a High-Intensity Green

Fluorescent Protein Fluorophage

Method for Drug- Resistance

Diagnosis in Tuberculosis for Isoniazid,

Rifampin, and Streptomycin.

Front. Microbiol. 7:922.

doi: 10.3389/fmicb.2016.00922
Xia Yu ${ }^{1}$, Yunting $\mathrm{Gu}^{1}$, Guanglu Jiang ${ }^{1}$, Yifeng $\mathrm{Ma}^{1}$, Liping Zhao ${ }^{1}$, Zhaogang Sun ${ }^{1}$, Paras Jain ${ }^{2}$, Max O'Donnell ${ }^{3,4}$, Michelle Larsen ${ }^{2}$, William R. Jacobs $\mathrm{Jr}^{2,5}$ and Hairong Huang ${ }^{1 *}$

${ }^{1}$ National Clinical Laboratory on Tuberculosis, Beijing Key Laboratory for Drug-Resistant Tuberculosis Research, Beijing Chest Hospital, Beijing Tuberculosis and Thoracic Tumor Institute, Capital Medical University, Beijing, China, ${ }^{2}$ Department of Microbiology and Immunology, Albert Einstein College of Medicine, Bronx, NY, USA, ${ }^{3}$ Division of Pulmonary, Allergy, and Critical Care Medicine, Columbia University Medical Center, New York, NY, USA, ${ }^{4}$ Department of Epidemiology, Mailman School of Public Health, Columbia University Medical Center, New York, NY, USA, ${ }^{5}$ Howard Hughes Medical Institute, Chevy Chase, MD, USA

A novel method for detecting drug resistance in Mycobacterium tuberculosis using mycobacteriophage $\Phi^{2}$ GFP10 was evaluated with clinical isolates. The phage facilitates microscopic fluorescence detection due to the high expression of green fluorescence protein which also simplifies the operative protocol as well. A total of 128 clinical isolates were tested by the phage assay for isoniazid $(\mathrm{INH})$, rifampin (RIF), and streptomycin (STR) resistance while conventional drug susceptibility test, by MGIT960, was used as reference. The sensitivities of $\Phi^{2}$ GFP10 assay for INH, RIF, and STR resistance detection were 100, 98.2, and 89.3\%, respectively while their specificities were 85.1, 98.6, and 95.8\%, respectively. The agreement between phage and conventional assay for detecting INH, RIF, and STR resistance was 92.2, 98.4, and 93.0\%, respectively. The $\Phi^{2}$ GFP10-phage results could be available in 2 days for RIF and STR, while it takes 3 days for $\mathrm{INH}$, with an estimated cost of less than $\$ 2$ to test all the three antibiotics. The $\Phi^{2}$ GFP10-phage method has the potential to be a valuable, rapid and economical screening method for detecting drug-resistant tuberculosis.

Keywords: tuberculosis, mycobacteriophage, drug resistance, diagnosis, $\Phi^{2}$ GFP10

\section{INTRODUCTION}

Reduction in transmission of drug resistant tuberculosis (TB) and improved patient management requires timely diagnosis of drug resistant bacilli. Presence of drug-resistant $M$. tuberculosis bacilli is confirmed by genotypic and phenotypic drug susceptibility testing (DST). Genotypic approaches with short turnaround time are based on identification of well-known antibiotic resistance-conferring gene mutations. Despite active research, not all TB drugs can have genotypic drug-susceptibility testing since the genetic basis of resistance may be complex or incompletely characterized (Kruuner et al., 2003; Jain et al., 2011; Cui et al., 2013; Zhang et al., 2015). Phenotypic 
assays observe the bacterial response to antibiotics in vitro without limitation to any particular antibiotic, allele, or working mechanism although the phenotypic DST is often time consuming. The conventional DST, based on solid medium, takes about 4-6 weeks after the isolation of $M$. tuberculosis, on the other hand BACTEC MGIT960 requires 10-14 days after acquiring a positive culture. Therefore, it is imperative to outline a further rapid, accurate, inexpensive DST method for the diagnosis of drug resistance $M$. tuberculosis.

Phage based methods have been used for drug resistance detection of $M$. tuberculosis since about two decades ago, such as bacteriophage D29 (Wilson et al., 1997) and luciferase reporter phages (LRPs) (Jacobs et al., 1993). A novel, high-intensity mycobacteria-specific fluorophage $\left(\Phi^{2} G F P 10\right)$ was described recently with good results in pre-clinical evaluation of drug resistant tuberculosis (Jain et al., 2012). $\Phi^{2}$ GFP10 is an improved second generation fluorescent reporter phage which expresses $g f p$ (green fluorescence protein ) under the control of $\mathrm{P}_{\mathrm{L}}$ promoter of mycobacterium phage L5 (Guo and Ao, 2012; Jain et al., 2012) and have intensity 100 times brighter than the previous generation of fluorescent reporter phages (Piuri et al., 2009). Unlike the LRPs (Jacobs et al., 1993), the $\Phi^{2} G F P 10$ reporter phage does not require exogenous luciferase substrate and can yield more stable and microscopically detectable intensive fluorescence. The presented study has evaluated the performance of this in-house fluorophage method to determine the rifampicin (RIF), isoniazid (INH), and streptomycin (STR) resistance in a high drug-resistant setting. Here we have evaluated the performance of $\Phi^{2} G F P 10$ phage for detecting drug resistance from $M$. tuberculosis clinical isolates, and also developed a new phage assay method using fluorescent microplate reader.

\section{MATERIALS AND METHODS}

\section{Strains}

A total of 128 clinical M. tuberculosis strains were isolated from patients with suspected drug-resistant TB patients visiting the Beijing chest hospital (Beijing, China) from April to June 2014. All of the isolates were identified as $M$. tuberculosis complex (MTBC) strains by performing a growth test on $500 \mu \mathrm{g} / \mathrm{ml}$ p-Nitrobenzoic Acid containing Löwenstein-Jensen medium (Tsukamura and Tsukamura, 1964). The drug susceptibility of the isolates was determined by MGIT960 SIRE kits according to the manufacturer's protocol. The critical concentrations used were as follows: INH $(0.1 \mu \mathrm{g} / \mathrm{ml}), \operatorname{RIF}(1.0 \mu \mathrm{g} / \mathrm{ml}), \operatorname{STR}(1.0 \mu \mathrm{g} / \mathrm{ml})$. Ultimately, 61, 56, and 56 were defined as resistant to INH, RIF and STR respectively by MGIT960 system. The laboratory $M$. tuberculosis H37Rv (ATCC 27294) strain was used in all batches as control.Technique round was demonstrated in Figure 1.

\section{Phage Stock Preparation}

The fluorophage, $\Phi^{2}$ GFP10 was constructed by Dr William R. Jacobs' laboratory in Albert Einstein University (New York, USA). The stocks were prepared by growing $M$. smegmatis strain $\left(\mathrm{mc}^{2} 155\right)$ in the $7 \mathrm{H} 9$, containing $10 \%$ oleic albumin dextrose catalase (OADC), to an optical density of 1.0 detected by spectrophotometer at $600 \mathrm{~nm}$ wavelength. Then $300 \mu \mathrm{l}$ cell suspension and $200 \mu \mathrm{l} \Phi^{2}$ GFP10 were mixed and incubated at room temperature for $30 \mathrm{~min}$. Subsequently, $3 \mathrm{ml} 7 \mathrm{H} 9$ containing $0.75 \%$ agar was added to the tube, the contents were briefly mixed and poured onto a 100-mm Petri dish containing 7H10-OADC. After the top-agar was solidified, the dish was incubated at $30^{\circ} \mathrm{C}$ for $48 \mathrm{~h}$. Then $3 \mathrm{ml} \mathrm{MP}$ buffer [ $50 \mathrm{mM}$ Tris ( $\mathrm{pH} 7.6), 150 \mathrm{mM}$ $\mathrm{NaCl}, 10 \mathrm{mM} \mathrm{MgCl}_{2}$, and $2 \mathrm{mM} \mathrm{CaCl}$ ] was pipetted onto the plates and then incubated on an orbital shaker for $6 \mathrm{~h}$. The buffer was then collected and passed through a $0.22 \mu \mathrm{m}$ Millipore filter membrane. The phage titers were determined by serial dilution method and the phage density was adjusted to approximately $10^{10}$ plaque forming unit $(\mathrm{pfu}) / \mathrm{ml}$ for further use. The phage stock was stable when stored at $4^{\circ} \mathrm{C}$ as no appreciable drop in titer occurred for at least 6 months.

\section{$\Phi^{2}$ GFP10 Assay by Microscopy Examination}

INH, RIF, and STR were obtained from Sigma-Aldrich. INH and STR were dissolved in water; RIF was dissolved in DMSO. The stock solutions were $20 \mathrm{mg} / \mathrm{ml}, 2 \mathrm{mg} / \mathrm{ml}$ and $4 \mathrm{mg} / \mathrm{ml}$ for INH, RIF, and STR, respectively. All stock solutions were stored at $-80^{\circ} \mathrm{C}$ during experimentation. Fresh colonies on LöwensteinJensen media were suspended and homogenized with sterile saline and the cell density was adjusted to $1 \mathrm{McF}$ arland turbidity. A $1 \mathrm{ml}$ aliquot was harvested by centrifugation and the sediment was re-suspended with $250 \mu \mathrm{l} 7 \mathrm{H} 9$-OADC in the absence of Tween 80 . The desired drugs would be added at this step, when needed, at the following finial concentrations: $20 \mu \mathrm{g} / \mathrm{ml}$ for INH, $2 \mu \mathrm{g} / \mathrm{ml}$ for RIF, or $4 \mu \mathrm{g} / \mathrm{ml}$ for STR. The tubes were incubated at $37^{\circ} \mathrm{C}$ for $24 \mathrm{~h}$, or $48 \mathrm{~h}$ specifically for INH tests. From the stock, containing $10^{10} \mathrm{pfu}$ fluorophage per $\mathrm{ml}, 100 \mu \mathrm{l}$ volume was added to each tube to obtain a multiplicity of infection (MOI) of 100 , the tubes were then incubated at $37^{\circ} \mathrm{C}$ for $16 \mathrm{~h}$. After adding $350 \mu \mathrm{l}$ of $4 \%$ paraformaldehyde in phosphate-buffered saline (PBS) to each tube, the reaction mixtures, left at room temperature for $90 \mathrm{~min}$ to ensure killing of the bacteria, were then centrifuged at $12,000 \mathrm{~g}$ for $15 \mathrm{~min}$ to remove excess phage and media. The pellet was re-suspended with $10 \mu \mathrm{l} 7 \mathrm{H} 9$ media. Five micro liter re-suspension was spotted on a glass slide and visualized by a fluorescence microscope. The criterion for resistance with phage assay was the presence of at least one fluorescent bacillus per 50 high power fields (Rondon et al., 2011).

\section{$\Phi^{2}$ GFP10 Assay by Microplate Reader}

To detect fluorescence of $\Phi^{2}$ GFP10 in a 96 well format relative light unit (RLU) of fluorescence was measured by a fluorescent microplate reader (Berthold LB970, Germany). The wavelength of excitation and emitted light was 485 and $535 \mathrm{~nm}$ respectively and the counting time was $5 \mathrm{~s}$. Each test was performed in triplicate and the mean value was used for interpretation. After removing excess phage and media by centrifuging, the pellet was re-suspended in $200 \mu \mathrm{l} \mathrm{PBS}$, and then, the RLU of the plate was read. The influence of drug exposure on the fluorescence production of the bacilli was interpreted by the remaining fluorescence rate (RFR), calculated by the following formula: (reaction counts-blank counts)/(positive control counts -blank counts) $\times 100 \%$ ("reaction" indicates the well with drug exposure; "blank" indicates the well without drug and phage; "positive control" indicates the reaction without drug). Precision 


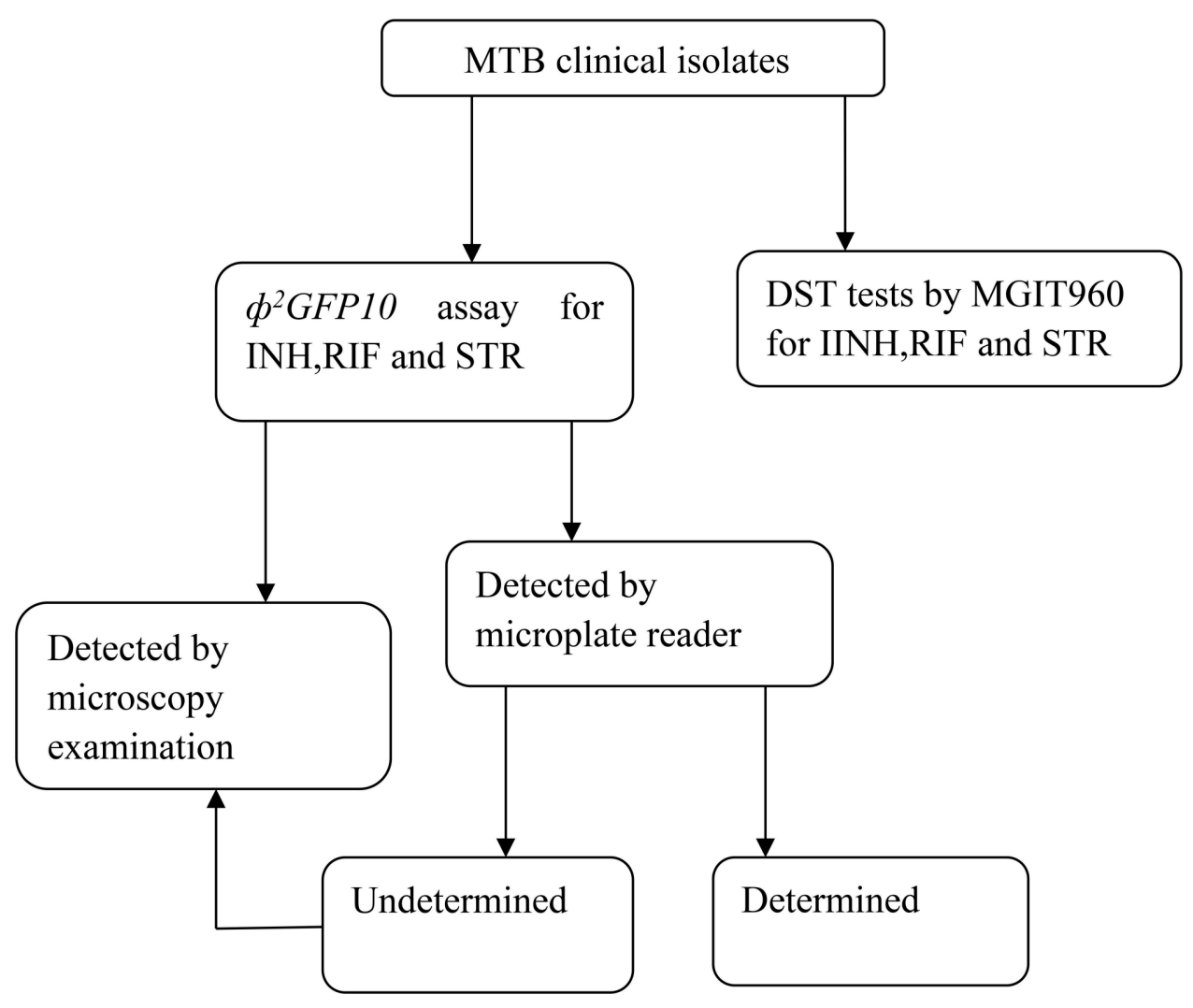

FIGURE 1 | Technique route of the study.

of the assay was determined with quintuple measurements of the following three strains: $\mathrm{H} 37 \mathrm{Rv}$ which was susceptible to all the three tested drugs, strain 14,301 which was resistant to all the three tested drugs and strain 14,161 which was resistant to INH and STR but susceptible to RIF. The stability of fluorescence was determined by detecting the same samples at 0,4 , and $24 \mathrm{~h}$, respectively. All the reactions were performed in triplicate.

\section{Statistical Analysis}

According to MGIT960 phenotypic outcomes, receiveroperation characteristic (ROC) analysis was performed using SPSS (version 17.0) and used to define a cutoff value for phage plate reader assay. Kappa value was calculated to compare the method between $\Phi^{2}$ GFP10 assay and MGIT960 phenotypic outcomes. Sensitivity, specificity, positive predictive value $(\mathrm{PPV})$, negative predictive value (NPV) was calculated from the following URL: http://vassarstats.net. The discriminative power of fluorescent microplate reader for drug resistance was analyzed using ROC curve and area under the curve (AUC). The optimal cut-off value was defined as the one with the least (1 sensitivity $)^{2}+\left(1-\right.$ specificity $^{2}$ value (Shu et al., 2013).

\section{RESULTS}

\section{$\Phi^{2}$ GFP10 Assay by Microscopy}

According to the outcomes of MGIT960, the sensitivity of $\Phi^{2}$ GFP10 assay for detecting INH, RIF and STR resistance were
$100,98.2$, and $89.3 \%$ respectively whereas the specificities were $85.1,98.6$, and $95.8 \%$ respectively. The agreement between phage assay and MGIT960 for detecting INH, RIF and STR resistance was 92.2, 98.4, and 93.0\%, respectively (see Table 1).The Kappa coefficient between $\Phi 2$ GFP10 assay and MGIT960 phenotypic outcomes for INH,RIF and STR were $0.85,0.97$, and 0.856 , respectively.

\section{$\Phi^{2}$ GFP10 Assay by Fluorescent Microplate Reader}

In the preliminary validation of the assay among the 3 tested isolates, the stability tests demonstrated that the fluorescence was stable for at least $24 \mathrm{~h}$ at $4{ }^{\circ} \mathrm{C}$ (see Table 2). The coefficient of variation $(\mathrm{CV})$ of RLU among the tested isolates in quintuple ranged from 1.22 to $6.73 \%$, with the mean value at $4.67 \pm 1.26 \%$. In the validation assays using clinical isolates, in contrast to the phenotypic DST, the AUC under the ROC curve of INH, RIF and STR were 0.957, 0.960, 0.917, and the optimal cutoff value for each drug has been listed in Table 3 and Figure 2. The sensitivity of $\Phi^{2}$ GFP10 assay by plate reader for detecting INH, RIF and STR resistance, according to the cutoff values, were 86.9, 89.3, and $83.9 \%$, respectively, while the specificity for INH, RIF and STR resistance detection were 94.0, 90.3, and 87.5\%, respectively. The Kappa coefficient between $\Phi^{2}$ GFP10 assay and MGIT960 phenotypic outcomes for INH,RIF and STR were 0.73, 0.79, and 0.71 ,respectively. Furthermore, evaluation of the RFR value 
TABLE 1 | Results and performance parameters: $\Phi^{2}$ GFP10 assay by microscopy vs. MGIT960 system.

\begin{tabular}{|c|c|c|c|c|c|c|c|c|c|}
\hline \multirow[t]{2}{*}{ Antibiotics } & \multicolumn{5}{|c|}{ No. of isolates } & \multicolumn{4}{|c|}{$\%(95 \% \mathrm{Cl})$} \\
\hline & Phage R & Phage S & Phage R & Phage S & Total & Sensitivity & Specificity & PPV & NPV \\
\hline $\mathrm{INH}$ & 61 & 0 & 10 & 57 & 128 & $100.0(92.6-100.0)$ & $85.1(73.8-92.2)$ & $85.9(75.2-92.7)$ & $100.0(92.1-100.0)$ \\
\hline RIF & 55 & 1 & 1 & 71 & 128 & $98.2(89.2-99.9)$ & 98.6 (91.5-99.9) & $98.2(89.2-99.9)$ & 198.6 (91.5-99.9) \\
\hline STR & 50 & 6 & 3 & 69 & 128 & $89.3(77.4-95.6)$ & $95.8(87.5-98.9)$ & $94.3(83.4-98.5)$ & $92.0(82.8-96.7)$ \\
\hline
\end{tabular}

$R$, resistant; S, sensitive.

TABLE 2 | The stability and precision of fluorescent microplate reader assay.

\begin{tabular}{lcccccc}
\hline Stains & Time $(\mathbf{h})$ & \multicolumn{4}{c}{ The counts of Fluorescent microplate reader (mean \pm SD) } \\
\cline { 2 - 6 } & & Blank control & Positive control & INH (RFR ${ }^{*}$ ) & RIF (RFR $\left.{ }^{*}\right)$ & STR (RFR $^{*}$ ) \\
\hline H37Rv & 0 & $5476.00 \pm 342.39$ & $9902.00 \pm 503.95$ & $6926.00 \pm 84.44(32.76)^{*}$ & $6296.00 \pm 267.82(18.53)$ & $6540.00 \pm 256.12(24.04)$ \\
& 4 & $5336.00 \pm 359.35$ & $9626.00 \pm 515.10$ & $6792.00 \pm 181.30(33.94)$ & $6974.00 \pm 323.77(26.53)$ & $6766.00 \pm 253.24(33.33)$ \\
& 24 & $5218.00 \pm 299.03$ & $10088.00 \pm 624.40$ & $6414.00 \pm 283.16(24.60)$ & $6364.00 \pm 335.01(23.53)$ & $6476.00 \pm 302.62(25.83)$ \\
\hline 14031 & 0 & $5144.00 \pm 184.07$ & $8650.00 \pm 347.20$ & $8058.00 \pm 297.19(83.11)$ & $8688.00 \pm 413.55(108.08)$ & $8314.00 \pm 533.32(90.42)$ \\
& 4 & $5140.00 \pm 252.59$ & $8410.00 \pm 312.17$ & $7992.00 \pm 342.15(87.22)$ & $8302.00 \pm 452.46(97.70)$ & $8352.00 \pm 448.74(98.23)$ \\
& 24 & $5318.00 \pm 332.60$ & $8558.00 \pm 257.04$ & $8230.00 \pm 278.57(89.88)$ & $8470.00 \pm 527.4(97.28)$ & $8516.00 \pm 310.85(98.70)$ \\
\hline 14161 & 0 & $4568.00 \pm 273.17$ & $8354.00 \pm 417.17$ & $7694.00 \pm 327.46(82.57)$ & $5380.00 \pm 185.07(21.45)$ & $7612.00 \pm 353.09(80.40)$ \\
& 4 & $4576.00 \pm 298.63$ & $8338.00 \pm 363.00$ & $7566.00 \pm 458.34(79.48)$ & $5768.00 \pm 206.57(31.69)$ & $7650.00 \pm 401.25(81.71)$ \\
& 24 & $4688.00 \pm 80.44$ & $8502.00 \pm 378.31$ & $7948.00 \pm 382.84(85.47)$ & $5320.00 \pm 257.97(16.57)$ & $7884.00 \pm 477.94(83.80)$
\end{tabular}

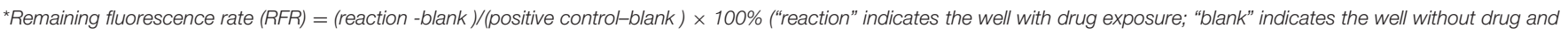
phage; "positive control" indicates the reaction without drug).

according to the susceptibility predictability demonstrated that when the RFR is lower than $45 \%$ the NPV for INH, RIF and STR were $98.0,98.2$, and $87.5 \%$, respectively. Similarly, when the ratio was higher than 60\%, the PPV for INH, RIF and STR were 92.6, 97.9 , and $95.3 \%$, respectively. When the ratio was between 45 and $60 \%$, then the outcome should be interpreted as undetermined (see Table 4).

\section{DISCUSSION}

Significant efforts are being made to develop rapid, simple and accurate tests for drug- resistant M. tuberculosis. One of such technologies currently been worked at is based on using bacteriophages (Banaiee et al., 2008; Piuri et al., 2009; Rondon et al., 2011; Jain et al., 2012; Sivaramakrishnan et al., 2013). LRP have been used for identifying drug resistance by detecting the luminescence produced by LRP after their infection in M. tuberculosis. These assays classify the samples as drugsusceptible if no luminescence is detected in the luminescence drug-containing samples. A meta-analysis showed that the sensitivity and specificity of luciferase reporter phage for rapid detection of RIF resistance in M. tuberculosis ranged from 92 to $100 \%$ and 89 to $100 \%$ (Minion and Pai, 2010). Although this method has shown high sensitivity and high specificity, a relatively expensive luminometer is required. The newly constructed phage $\Phi^{2}$ GFP10 facilitates the microscopic signal detection since it yields 100 -folds higher fluorescence signal percell than any previously described reporter phages (Jain et al.,
2011). Meanwhile, as the intensive fluorescence increased the contrast between reaction and background, tedious washing steps were not necessary for phage assay, so the $\Phi^{2} G F P 10$ assay was easier to perform than the other phage assays (Rondon et al., 2011).

In this study, drug resistance detection by $\Phi^{2}$ GFP10 assay yielding applicable sensitivities and specificities, especially for RIF. A recent report also obtained great consistency between $\Phi^{2}$ GFP10 assay and GeneXpert for RIF resistance diagnosis (O’Donnell et al., 2015). Fluorescent microscopy may be a limiting factor for $\Phi^{2} G F P 10$ assay application due to its cost, whereas less expensive and simpler fluorescent microscopes using light emitting diodes (LED) have been applied for smear test of tuberculosis in various clinical laboratories (Marzouk et al., 2013; Reza et al., 2013; Xia et al., 2013) and they could readily be adapted for $\Phi^{2}$ GFP10 assay. The reagents for fluorophage growth and amplification are inexpensive, safe, and universally available. The total cost for the 3-drug test per strain was less than US \$2. Additionally, fixation of the sample with paraformaldehyde prior to analysis overcomes the substantial biosafety concerns.

In our study, even though a longer drug exposure time of $48 \mathrm{~h}$ for INH, the specificity was still lower than those of RIF and STR whose drug exposure time was $24 \mathrm{~h}$. Those observations were consistent with other mycobacteriophage-based assays (Rondon et al., 2011). The anti-TB mechanism of INH involves inhibiting the synthesis of mycolic acid of cell wall (Eltringham et al., 1999), which is a slow process compared with the quickly bactericidal activities of RIF and STR, therefore the bacilli can stay alive 
TABLE 3 | Results and performance parameters: fluorescent microplate reader vs. MGIT960 system.

\begin{tabular}{|c|c|c|c|c|c|c|}
\hline \multirow[t]{2}{*}{ Antibiotics } & \multirow[t]{2}{*}{ AUC } & \multirow[t]{2}{*}{ Cutoff (\%) } & \multicolumn{4}{|c|}{$\%(95 \% \mathrm{Cl})$} \\
\hline & & & sensitivity & specificity & PPV & NPV \\
\hline $\mathrm{INH}$ & 0.957 & 55.00 & 86.9 (75.2-93.8) & $94.0(84.7-98.1)$ & $93.0(82.2-97.7)$ & 88.7 (78.5-94.7) \\
\hline RIF & 0.960 & 53.60 & 89.3 (77.4-95.6) & $90.3(80.4-95.7)$ & 87.7 (75.7-94.5) & 91.5 (81.9-96.5) \\
\hline STR & 0.917 & 48.65 & 83.9 (71.2-91.9) & 87.5 (77.1-93.8) & 83.9 (71.2-91.9) & 87.5 (77.1-93.8) \\
\hline
\end{tabular}
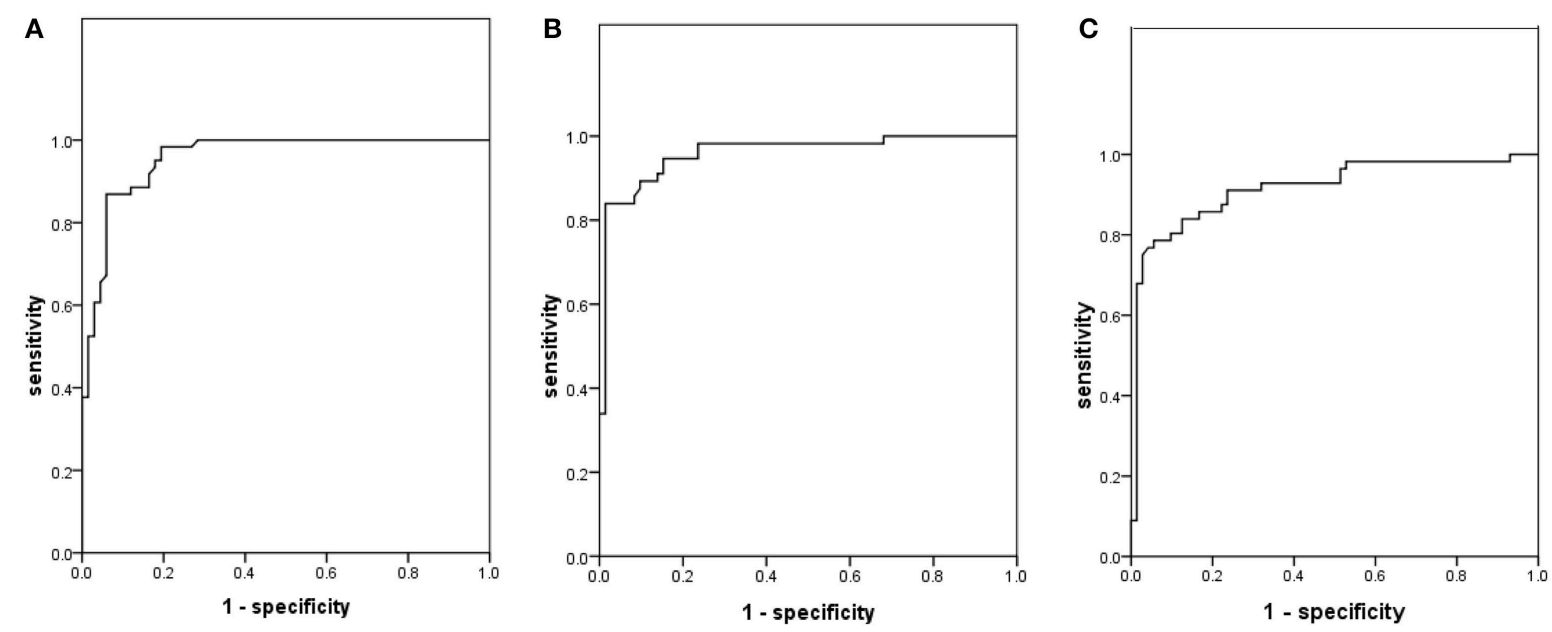

FIGURE 2 | Receiver operating characteristic curves of fluorescent microplate reader in detecting drug resistance according to BactecMGIT 960 system. (A) INH; (B) RIF; (C) STR.

TABLE 4 | Results and performance parameters by sectional outcomes interpretation: fluorescent microplate reader vs. MGIT960 system.

\begin{tabular}{lcccc}
\hline \multirow{2}{*}{ Drug } & RFR & No. of isolates & \multicolumn{2}{c}{ MGIT960 DST outcomes } \\
\cline { 3 - 5 } & & & $\mathbf{R}$ & $\mathbf{S}$ \\
\hline $\mathrm{INH}$ & $<45 \%$ & 51 & 1 & 50 \\
& $45-60 \%$ & 23 & 10 & 13 \\
& $>60 \%$ & 54 & 50 & 4 \\
\hline RIF & $<45 \%$ & 56 & 1 & 55 \\
& $45-60 \%$ & 25 & 9 & 16 \\
& $>60 \%$ & 47 & 46 & 1 \\
\hline STR & $<45 \%$ & 64 & 8 & 56 \\
& $45-60 \%$ & 21 & 7 & 14 \\
\hline
\end{tabular}

$R$, resistant; $S$, sensitive; RFR, remaining fluorescence rate.

for quite a while even when they are sensitive to INH. The fluorophage could infect such bacilli and produce fluorescence signal which decreased the specificity of the assay. To enhance the bactericidal activity of INH, we increased the INH concentration for phage assay to $20 \mu \mathrm{g} / \mathrm{ml}$, which was dramatically higher than those of previous studies (Xia et al., 2013; Zhang et al., 2015). However, the specificity was still lower for INH than those of RIF and STR, which indicating the INH tolerance deviation for clinical strains was universal.
First-line-drug ethambutol (EMB) also affects the cell wall construction in M. tuberculosis, and is a bacteriostatic drug rather than a bactericidal drug. We attempted to develop a $\Phi^{2}$ GFP10 assay for EMB, but the pilot test demonstrated that even an exposure concentration of $50 \mu \mathrm{g} / \mathrm{ml}$ could not lead to obvious CFU loss. A previous study reported low concordance (53\%) between the phage amplified biologically assay and resistance ratio method for $\mathrm{EMB}$, which they attributed to the bacteriostatic role of EMB both in vitro and in a macrophage model (Eltringham et al., 1999). Therefore, the $\Phi^{2} G F P 10$ assay might only be feasible to test the rapid-action bacteriocidal drugs.

Although we acquired favorable sensitivity and specificity for $\Phi 2 G F P 10$ assay by microscopy, we found that microscopic examination process is experience dependent, and a lot of time is required when handing a batch of samples. So we developed a phage assay to interpret the outcomes simply by fluorescence plate reader, which can read a 96-well plate in $10 \mathrm{~min}$. We monitored the fluorescence diminishing after the bacilli were exposed to the tested drugs. When categorized the RFR lower than $45 \%$ as "susceptible," greater than $60 \%$ as "resistant," while between 45 and $60 \%$ as undetermined, plate reader could interpret over $80 \%$ of the test strains successfully for all the 3 drugs, with 95.24\% (100/105), 98.06\% (101/103), and $90.65 \%(97 / 107)$ consistency with MGIT960 system for INH, RIF, and STR susceptibility tests, respectively. Therefore, we suggest interpreting the outcomes in sections to increase the reliability of the assay. For undetermined samples, we 
suggest to recheck by microscopy to produce more reliable results.

Like other fluoromycobacteriophages, $\Phi^{2} G F P 10$ assay could not discriminate between $M$. tuberculosis and other members of MTBC species (Riska et al., 1997). For the settings with high isolation rate of nontuberculous mycobacteria, one should be cautious to report drug resistance without MTBC identification.

In summary, the $\Phi^{2}$ GFP10 assay is a rapid, simple and inexpensive DST method. In clinical laboratories with limited resources, the $\Phi^{2}$ GFP10 based assay has the potential to be used for drug resistance diagnosis of tuberculosis.

\section{AUTHOR CONTRIBUTIONS}

$\mathrm{HH}$ designed the study. $\mathrm{HH}$ and $\mathrm{XY}$ wrote the paper. WJ modified the paper. XY and YG performed the $\Phi^{2} G F P 10$

\section{REFERENCES}

Banaiee, N., January, V., Barthus, C., Lambrick, M., Roditi, D., Behr, M. A., et al. (2008). Evaluation of a semi-automated reporter phage assay for susceptibility testing of Mycobacterium tuberculosis isolates in South Africa. Tuberculosis (Edinb) 88, 64-68. doi: 10.1016/j.tube.2007.08.006

Cui, Z., Wang, J., Lu, J., Huang, X., Zheng, R., and Hu, Z. (2013). Evaluation of methods for testing the susceptibility of clinical Mycobacterium tuberculosis isolates to pyrazinamide. J. Clin. Microbiol. 51, 1374-1380. doi: 10.1128/jcm.03197-12

Eltringham, I. J., Wilson, S. M., and Drobniewski, F. A. (1999). Evaluation of a bacteriophage-based assay (phage amplified biologically assay) as a rapid screen for resistance to isoniazid, ethambutol, streptomycin, pyrazinamide, and ciprofloxacin among clinical isolates of Mycobacterium tuberculosis. J. Clin. Microbiol. 37, 3528-3532.

Guo, S., and Ao, Z. (2012). Phage in the diagnosis and treatment of tuberculosis. Front. Biosci. 17, 2691-2697. doi: 10.2741/4080

Jacobs, W. R. Jr., Barletta, R. G., Udani, R., Chan, J., Kalkut, G., Sosne, G., et al. (1993). Rapid assessment of drug susceptibilities of Mycobacterium tuberculosis by means of luciferase reporter phages. Science 260, 819-822. doi: $10.1126 /$ science. 8484123

Jain, P., Hartman, T. E., Eisenberg, N., O’Donnell, M. R., Kriakov, J., Govender, K., et al. (2012). phi(2)GFP10, a high-intensity fluorophage, enables detection and rapid drug susceptibility testing of Mycobacterium tuberculosis directly from sputum samples. J. Clin. Microbiol. 50, 1362-1369. doi: 10.1128/jcm.06192-11

Jain, P., Thaler, D. S., Maiga, M., Timmins, G. S., Bishai, W. R., Hatfull, G. F., et al. (2011). Reporter phage and breath tests: emerging phenotypic assays for diagnosing active tuberculosis, antibiotic resistance, and treatment efficacy. J. Infect. Dis. 204(suppl. 4), S1142-S1150. doi: 10.1093/infdis/jir454

Kruuner, A., Jureen, P., Levina, K., Ghebremichael, S., and Hoffner, S. (2003). Discordant resistance to kanamycin and amikacin in drug-resistant Mycobacterium tuberculosis. Antimicrob. Agents Chemother. 47, 2971-2973. doi: 10.1128/AAC.47.9.2971-2973.2003

Marzouk, M., Ferjani, A., Dhaou, M., Ali, M. H., Hannachi, N., and Boukadida, J. (2013). Comparison of LED and conventional fluorescence microscopy for detection of acid-fast bacilli in an area with high tuberculosis incidence. Diagn. Microbiol. Infect. Dis. 76, 306-308. doi: 10.1016/j.diagmicrobio.2013.03.023

Minion, J., and Pai, M. (2010). Bacteriophage assays for rifampicin resistance detection in Mycobacterium tuberculosis: updated meta-analysis. Int. J. Tuberc. Lung. Dis. 14, 941-951.

O’Donnell, M. R., Pym, A., Jain, P., Munsamy, V., Wolf, A., Karim, F., et al. (2015). A novel reporter phage to detect tuberculosis and rifampin resistance in a high-HIV-burden population. J. Clin. Microbiol. 53, 2188-2194. doi: 10.1128/jcm.03530-14

Piuri, M., Jacobs, W. R. Jr., and Hatfull, G. F. (2009). Fluoromycobacteriophages for rapid, specific, and sensitive antibiotic susceptibility testing of Mycobacterium tuberculosis. PLoS ONE 4:e4870. doi: 10.1371/journal.pone.0004870 assay by microscopy. XY and ZS performed $\Phi^{2} G F P 10$ assay by Microplate reader. GJ, YM, and LZ performed DST by MGIT960 kits. XY conducted statistical analysis. PJ, MO, and ML established the assay for phage stock preparation. All authors reviewed the results and approved the final version of the manuscript.

\section{ACKNOWLEDGMENTS}

The work was supported by the research funding from Infectious Diseases Special Project, Minister of Health of China (2012ZX10003002-009, 2016ZX1000300112), The capital health research and development of special (2016-4-1042). WJ is supported by the National Institute of Health (AI26170). ML is supported by NIH (1R01AI114900).

Reza, L. W., Satyanarayna, S., Enarson, D. A., Kumar, A. M., Sagili, K., Kumar, S., et al. (2013). LED-fluorescence microscopy for diagnosis of pulmonary tuberculosis under programmatic conditions in India. PLOS ONE 8:e75566. doi: 10.1371/journal.pone.0075566

Riska, P. F., Jacobs, W. R. Jr., Bloom, B. R., McKitrick, J., and Chan, J. (1997). Specific identification of Mycobacterium tuberculosis with the luciferase reporter mycobacteriophage: use of p-nitro-alpha-acetylamino-beta-hydroxy propiophenone. J Clin Microbiol 35, 3225-3231.

Rondon, L., Piuri, M., Jacobs, W. R. Jr., De Waard, J., Hatfull, G. F., and Takiff, H. E. (2011). Evaluation of fluoromycobacteriophages for detecting drug resistance in Mycobacterium tuberculosis. J. Clin. Microbiol. 49, 1838-1842. doi: 10.1128/jcm.02476-10

Shu, C. C., Wu, M. F., Hsu, C. L., Huang, C. T., Wang, J. Y., Hsieh, S. L., et al. (2013). Apoptosis-associated biomarkers in tuberculosis: promising for diagnosis and prognosis prediction. BMC Infect. Dis. 13:45. doi: 10.1186/1471-2334-13-45

Sivaramakrishnan, G., Subramanyam, B., C, P., and Kumar, V. (2013). Luciferase reporter phage phAE85 for rapid detection of rifampicin resistance in clinical isolates of Mycobacterium tuberculosis. Asian Pac. J. Trop. Med. 6, 728-731. doi: 10.1016/s1995-7645(13)60127-3

Tsukamura, M., and Tsukamura, S. (1964). Differentiation of Mycobacterium tuberculosis and Mycobacterium bovis by p-nitrobenzoic acid susceptibility. Tubercle 45, 64-65. doi: 10.1016/S0041-3879(64)80091-X

Wilson, S. M., Al-Suwaidi, Z., McNerney, R., Porter, J., and Drobniewski, F. (1997). Evaluation of a new rapid bacteriophage-based method for the drug susceptibility testing of Mycobacterium tuberculosis. Nat. Med. 3, 465-468. doi: 10.1038/nm0497-465

Xia, H., Song, Y. Y., Zhao, B., Kam, K. M., O’Brien, R. J., Zhang, Z. Y., et al. (2013). Multicentre evaluation of Ziehl-Neelsen and light-emitting diode fluorescence microscopy in China. Int. J. Tuberc. Lung. Dis. 17, 107-112. doi: 10.5588/ijtld.12.0184

Zhang, X., Liu, L., Zhang, Y., Dai, G., Huang, H., and Jin, Q. (2015). Genetic determinants involved in p-aminosalicylic acid resistance in clinical isolates from tuberculosis patients in northern China from 2006 to 2012. Antimicrob. Agents Chemother. 59, 1320-1324. doi: 10.1128/aac. 03695-14

Conflict of Interest Statement: The authors declare that the research was conducted in the absence of any commercial or financial relationships that could be construed as a potential conflict of interest.

Copyright (C) 2016 Yu, Gu, Jiang, Ma, Zhao, Sun, Jain, O'Donnell, Larsen, Jacobs and Huang. This is an open-access article distributed under the terms of the Creative Commons Attribution License (CC BY). The use, distribution or reproduction in other forums is permitted, provided the original author(s) or licensor are credited and that the original publication in this journal is cited, in accordance with accepted academic practice. No use, distribution or reproduction is permitted which does not comply with these terms. 